\title{
Regret Theory and the Competitive Firm Revisited *
}

\author{
Udo Broll \\ Technische Universität Dresden \\ Peter Welzel \\ University of Augsburg \\ and \\ Kit Pong Wong \\ University of Hong Kong
}

\begin{abstract}
This paper revisits the impact of regret aversion on the behavior of the competitive firm under price uncertainty. We show that the firm optimally produces more (less) when regret aversion prevails if the random output price is positively (negatively) skewed. In this case, high (low) output prices are much more likely to be seen than low (high) output prices. To avoid regret, the firm is induced to raise (lower) its output optimal level. The skewness of the price distribution as such plays a pivotal role in determining how regret aversion affects the firm's production decision.
\end{abstract}

JEL classification: D21; D24; D81

Keywords: Production; Regret theory; Uncertainty

${ }^{*}$ Correspondence to: Udo Broll, Department of Business and Economics; School of International Studies (ZIS), Technische Universität Dresden, 01062 Dresden, Germany, e-mail: udo.broll@tu-dresden.de (U. Broll). 


\section{Introduction}

Individuals and firms have desires to avoid consequences wherein ex-post suboptimal decisions appear to have been made even though these decisions are ex-ante optimal based on the information available at that time. Taking this into consideration, Bell $(1982,1983)$ and Loomes and Sugden (1982) develop regret theory that defines regret as the disutility arising from not having chosen the ex-post optimal alternative, which is later axiomatized by Quiggin (1994) and Sugden (1993). Indeed, there is an extensive experimental literature that documents pervasive regret-averse preferences among individuals and firms (Loomes, 1988; Loomes et al., 1992; Loomes and Sugden, 1987; Starmer and Sugden, 1993).

Wong (2014) has recently incorporated regret theory into Sandmo's (1971) model of the competitive firm under price uncertainty. ${ }^{1}$ To this end, Wong (2014) characterizes the firm's regret-averse preferences by a modified utility function that includes additive separable disutility from having chosen ex-post suboptimal alternatives. ${ }^{2}$ The extent of regret depends on the difference between the actual profit and the maximum profit attained by making the optimal production decision had the firm observed the true realization of the random output price.

In this paper, we revisit Wong's (2014) model by deriving sufficient conditions under which regret aversion has unambiguous effect on the firm's production decision. We show that the firm optimally produces more (less) when regret aversion prevails if the random output price is positively (negatively) skewed. In this case, high (low) output prices are much more likely to be seen than low (high) output prices. To avoid regret, the firm is induced to raise (lower) its output optimal level. The skewness of the price distribution as such plays a pivotal role in determining how regret aversion affects the firm's production decision, which is a novel result in the literature.

\footnotetext{
${ }^{1}$ Wong (2014) is closely related to the early work of Paroush and Venezia (1979) who examine the competitive firm under uncertainty with a bivariate utility function defined on profits and regret.

${ }^{2}$ Other applications of regret theory include Braun and Muermann (2004), Broll et al. (2015), Muermann et al. (2006), and Wong (2011, 2012, 2015).
} 
The rest of this paper is organized as follows. Section 2 delineates the model of the competitive firm under uncertainty when the firm's preferences exhibit regret aversion. Section 3 examines the effect of regret aversion on the firm's optimal output level. The final section concludes.

\section{The model}

Consider the single-period model of the competitive firm under price uncertainty $\grave{a}$ la Sandmo (1971). At the beginning of the period, the firm produces a single commodity according to a deterministic cost function, $C(Q)$, where $Q \geq 0$ is the output level, and $C(Q)$ is compounded to the end of the period. Since the firm's production technology exhibits decreasing returns to scale, the cost function has the properties that $C(0)=C^{\prime}(0)=0$, and $C^{\prime}(Q)>0$ and $C^{\prime \prime}(Q)>0$ for all $Q>0$.

At the end of the period, the firm sells its entire output, $Q$, at the then prevailing perunit price, $\tilde{P}$, which is unknown ex ante. ${ }^{3}$ The uncertain per-unit price, $\tilde{P}$, is distributed according to a known cumulative distribution function $(\mathrm{CDF}), F(P)$, over support $[\underline{P}, \bar{P}]$, where $0<\underline{P}<\bar{P}$. ${ }^{4}$ The firm's end-of-period profit as a function of $P$ is given by

$$
\Pi(P)=P Q-C(Q)
$$

for all $P \in[\underline{P}, \bar{P}]$.

We assume that the firm's preferences are represented by the following "modified" utility function that includes some compensation for regret:

$$
U(\Pi)=\Pi-\beta G(R),
$$

\footnotetext{
${ }^{3}$ Throughout the paper, random variables have a tilde $(\sim)$ while their realizations do not.

${ }^{4} \mathrm{An}$ alternative way to model the price uncertainty is to apply the concept of information systems that are conditional cumulative distribution functions over a set of signals imperfectly correlated with $\tilde{P}$ (Broll et al., 2014).
} 
where $\beta>0$ is a positive constant, and $G(R)$ is a regret function defined over the magnitude of regret, $R$, such that $G(0)=0$, and $G^{\prime}(R)>0$ and $G^{\prime \prime}(R)>0$ for all $R>0 .{ }^{5}$ The magnitude of regret, $R=\Pi^{\max }-\Pi$, is measured by the difference between the actual profit, $\Pi$, and the maximum profit, $\Pi^{\max }$, that the firm could have earned at the end of the period should the firm have made the optimal production decision based on knowing the true per-unit price, $P$. Since $\Pi$ cannot exceed $\Pi^{\max }$, the firm experiences disutility from forgoing the possibility of undertaking the ex-post optimal production decision. The parameter, $\beta$, is a constant regret coefficient that reflects the increasing importance of regret aversion in representing the firm's preferences as $\beta$ increases.

To characterize the regret-averse firm's optimal production decision, we have to first determine the maximum profit, $\Pi^{\max }$. If the firm could have observed the true per-unit price, $P$, the maximum profit would be achieved if the firm had chosen $Q(P)$, which is the solution to $C^{\prime}[Q(P)]=P$. The maximum profit as a function of $P$ is, therefore, given by

$$
\Pi^{\max }(P)=P Q(P)-C[Q(P)]
$$

for all $P \in[\underline{P}, \bar{P}]$. Using Eqs. (1) and (3), we can write the magnitude of regret, $R(P)$, as

$$
R(P)=\Pi^{\max }(P)-\Pi(P)=P Q(P)-C[Q(P)]-[P Q-C(Q)],
$$

for all $P \in[\underline{P}, \bar{P}]$.

We can now state the regret-averse firm's ex-ante decision problem. At the beginning of the period, the firm chooses an output level, $Q$, so as to maximize the expected value of its regret-theoretical utility function defined in Eq. (2):

$$
\max _{Q \geq 0} \mathrm{E}\{\Pi(\tilde{P})-\beta G[R(\tilde{P})]\}
$$

where $\mathrm{E}(\cdot)$ is the expectation operator with respect to the cumulative distribution function, $F(P)$, and $\Pi(P)$ and $R(P)$ are given by Eqs. (1) and (4), respectively. The first-order

\footnotetext{
${ }^{5}$ Bleichrodt et al. (2010) provide empirical evidence that regret functions are indeed convex.
} 
condition for program (5) is given by

$$
\mathrm{E}(\tilde{P})-C^{\prime}\left(Q^{*}\right)+\beta \mathrm{E}\left\{G^{\prime}\left[R^{*}(\tilde{P})\right]\left[\tilde{P}-C^{\prime}\left(Q^{*}\right)\right]\right\}=0,
$$

where $Q^{*}$ is the optimal output level, and $R^{*}(P)=P Q(P)-C[Q(P)]-\left[P Q^{*}-C^{*}\left(Q^{*}\right)\right]$.

Differentiating $\mathrm{E}\{U[\Pi(\tilde{P})]\}$ twice with respect to $Q$ yields

$$
\frac{\partial^{2} \mathrm{E}\{U[\Pi(\tilde{P})]\}}{\partial Q^{2}}=-\beta \mathrm{E}\left\{G^{\prime \prime}[R(\tilde{P})]\left[\tilde{P}-C^{\prime}(Q)\right]^{2}\right\}-\mathrm{E}\left\{1+\beta G^{\prime}[R(\tilde{P})]\right\} C^{\prime \prime}(Q)<0
$$

for all $Q>0$, where the inequality follows from the properties of $G(R)$ and $C(Q)$. Eq. (7) implies that Eq. (6) is both necessary and sufficient for $Q^{*}$ to be the unique optimal solution to program (5).

\section{Regret aversion and production}

As a benchmark, suppose that the firm is not regret averse in that $\beta=0$. It then follows from Eq. (6) with $\beta=0$ that the optimal output level, $Q^{\circ}$, in this benchmark case solves

$$
\mathrm{E}(\tilde{P})-C^{\prime}\left(Q^{\circ}\right)=0
$$

To examine the impact of regret aversion on the firm's production decision, we compare

$Q^{*}$ with $Q^{\circ}$. To this end, we differentiate $\mathrm{E}\{U[\Pi(\tilde{P})]\}$ with respect to $Q$, and evaluate the resulting the derivative at $Q=Q^{\circ}$ to yield

$$
\left.\frac{\partial \mathrm{E}\{U[\Pi(\tilde{P})]\}}{\partial Q}\right|_{Q=Q^{\circ}}=\beta \mathrm{E}\left\{G^{\prime}\left[R^{\circ}(\tilde{P})\right][\tilde{P}-\mathrm{E}(\tilde{P})]\right\}
$$

where we have used Eq. (8), and $R^{\circ}(P)=P Q(P)-C[Q(P)]-\left[P Q^{\circ}-C\left(Q^{\circ}\right)\right]$ for all $P \in[\underline{P}, \bar{P}]$. It then follows from Eqs. (6) and (7) that $Q^{*}>(<) Q^{\circ}$ if, and only if, the right-hand side of Eq. (9) is positive (negative).

To gain more insights, we consider an example in which the regret function is quadratic, i.e., $G(R)=a R+b R^{2}$, where $a$ and $b$ are positive constants. We further assume that the 
cost function is also quadratic, $C(Q)=c Q^{2}$, where $c$ is a positive constant. In this example, we have $Q(P)=P / 2 c$ and $R(P)=[P-\mathrm{E}(\tilde{P})]^{2} / 4 c$. Hence, Eq. (9) becomes

$$
\left.\frac{\partial \mathrm{E}\{U[\Pi(\tilde{P})]\}}{\partial Q}\right|_{Q=Q^{\circ}}=\frac{b \beta}{2 c} \mathrm{E}\left\{[\tilde{P}-\mathrm{E}(\tilde{P})]^{3}\right\} .
$$

It then follows from Eq. (10) that $Q^{*}>(<) Q^{\circ}$ if, and only if, the third central moment, $\mathrm{E}\left\{[\tilde{P}-\mathrm{E}(\tilde{P})]^{3}\right\}$, is positive (negative). Hence, if $\tilde{P}$ is symmetrically distributed, we have $Q^{*}=Q^{\circ}$. On the other hand, if $\tilde{P}$ is positively (negatively) skewed, we have $Q^{*}>(<) Q^{\circ}$.

Motivated by the above example, we can look for a critical CDF of $\tilde{P}$, denoted by $F^{\circ}(P)$, such that $\tilde{P}$ has the same mean with respect to $F(P)$ and $F^{\circ}(P)$, and that $Q^{*}=Q^{\circ}$. Thus, we have $\int_{\underline{P}}^{\bar{P}} F(P) \mathrm{d} P=\int_{\underline{P}}^{\bar{P}} F^{\circ}(P) \mathrm{d} P$ and

$$
\int_{\underline{P}}^{\bar{P}} G^{\prime}\left[R^{\circ}(P)\right][P-\mathrm{E}(\tilde{P})] \mathrm{d} F^{\circ}(P)=0 .
$$

We say that $F(P)$ has more simple positive (negative) skewness than $F^{\circ}(P)$ if, and only if, $\int_{\underline{P}}^{P}\left[F(x)-F^{\circ}(x)\right] \mathrm{d} x \leq(\geq) 0$ for all $P \leq \mathrm{E}(\tilde{P})$ and $\int_{\underline{P}}^{P}\left[F(x)-F^{\circ}(x)\right] \mathrm{d} x \geq(\leq) 0$ for all $P \geq \mathrm{E}(\tilde{P})$. To see this, note that

$$
\int_{\underline{P}}^{\bar{P}}[P-\mathrm{E}(\tilde{P})]^{3} \mathrm{~d}\left[F(P)-F^{\circ}(P)\right]=6 \int_{\underline{P}}^{\bar{P}}[P-\mathrm{E}(\tilde{P})]\left\{\int_{\underline{P}}^{P}\left[F(x)-F^{\circ}(x)\right] \mathrm{d} x\right\} \mathrm{d} P,
$$

where the equality follows from integration by parts and $\int_{\underline{P}}^{\bar{P}} F(P) \mathrm{d} P=\int_{\underline{P}}^{\bar{P}} F^{\circ}(P) \mathrm{d} P$. If $F(P)$ has more simple positive (negative) skewness than $F^{\circ}(P)$, the right-hand side of Eq. (12) is positive (negative) so that the third central moment is larger (smaller) with respect to $F(P)$ than with respect to $F^{\circ}(P)$.

We state and prove our main result.

Proposition 1. If $G^{\prime \prime \prime}(R) \geq 0$, a sufficient condition that ensures the firm to increase (decrease) its optimal output level, i.e., $Q^{*}>(<) Q^{\circ}$, when regret aversion prevails is that the uncertain per-unit price, $\tilde{P}$, has a $C D F, F(P)$, that has more simple positive (negative) skewness than the critical $C D F, F^{\circ}(P)$. 
Proof. Let $H(P)=G^{\prime}\left[R^{\circ}(P)\right][P-\mathrm{E}(\tilde{P})]$. Then, we have

$$
\begin{aligned}
H^{\prime \prime}(P)= & G^{\prime \prime \prime}\left[R^{\circ}(P)\right]\left[Q(P)-Q^{\circ}\right]^{2}[P-\mathrm{E}(\tilde{P})] \\
& +G^{\prime \prime}\left[R^{\circ}(P)\right][P-\mathrm{E}(\tilde{P})] Q^{\prime}(P)+2 G^{\prime \prime}\left[R^{\circ}(P)\right]\left[Q(P)-Q^{\circ}\right] .
\end{aligned}
$$

Since $Q(P)<(>) Q^{\circ}$ whenever $P<(>) \mathrm{E}(\tilde{P})$ and $Q^{\prime}(P)=1 / C^{\prime \prime}[Q(P)]>0$, Eq. (13) implies that $H^{\prime \prime}(P)<(>) 0$ whenever $P<(>) \mathrm{E}(\tilde{P})$. Using integration by parts twice and Eq. (11), we can write the right-hand side of Eq. (9) as

$$
\beta \int_{\underline{P}}^{\bar{P}} H(P) \mathrm{d}\left[F(P)-F^{\circ}(P)\right]=\beta \int_{\underline{P}}^{\bar{P}} H^{\prime \prime}(P)\left\{\int_{\underline{P}}^{P}\left[F(x)-F^{\circ}(x)\right] \mathrm{d} x\right\} \mathrm{d} P,
$$

since $\int_{\underline{P}}^{\bar{P}} F(P) \mathrm{d} P=\int_{\underline{P}}^{\bar{P}} F^{\circ}(P) \mathrm{d} P$. If $F(P)$ has more simple positive (negative) skewness than $F^{\circ}(P)$, the right-hand side of Eq. (14) is positive (negative) so that $Q^{*}>(<) Q^{\circ}$.

The intuition for Proposition 1 is as follows. When $F(P)$ has more simple positive skewness than $F^{\circ}(P)$, realizations of $\tilde{P}$ close to $\underline{P}$ are much less likely to be seen than those close to $\bar{P}$. Introducing regret aversion to the firm makes the firm concern more about the disutility from the discrepancy of its output level, $Q(P)-Q^{n}$, when high realizations of $\tilde{P}$ are revealed. To avoid regret, the regret-averse firm optimally adjusts its output level upward from $Q^{n}$ so that $Q^{*}>Q^{n}$. On the other hand, when $F(P)$ has more simple negative skewness than $F^{\circ}(P)$, realizations of $\tilde{P}$ close to $\underline{P}$ are much more likely to be seen than those close to $\bar{P}$. The regret-averse firm as such optimally adjusts its output level downward from $Q^{n}$ to reduce the discrepancy of its output level, $Q^{n}-Q(P)$, when low output prices are revealed so that $Q^{*}<Q^{n}$.

\section{Conclusion}

In this paper, we revisit the regret-theoretical model of the competitive firm under price uncertainty as recently developed by Wong (2014). Regret-averse preferences are character- 
ized by a modified utility function that includes additive separable disutility from having chosen ex-post suboptimal alternatives. The extent of regret depends on the difference between the actual profit and the maximum profit attained by making the optimal production decision had the firm observed the true realization of the random output price. We show that the firm optimally produces more (less) when regret aversion prevails if the random output price is positively (negatively) skewed. In this case, high (low) output prices are much more likely to be seen than low (high) output prices. To avoid regret, the firm is induced to raise (lower) its output optimal level. This paper as such offers more general sufficient conditions under which regret aversion has unambiguous effect on the firm's production decision.

\section{References}

Bell, D. E., 1982. Regret in decision making under uncertainty. Operations Research 30, $961-981$.

Bell, D. E., 1983. Risk premiums for decision regret. Management Science 29, 1156-1166.

Bleichrodt, H., Cillo, A., Diecidue, E., 2010. A quantitative measurement of regret theory. Management Science 56, 161-175.

Braun, M., Muermann, A., 2004. The impact of regret on the demand for insurance. Journal of Risk and Insurance 71, 737-767.

Broll, U., Eckwert, B., Wong, K. P., 2014. Transparency and risk sharing in international trade. The Manchester School 82, 716-731.

Broll, U., Welzel, P., Wong, K. P., 2015. Exchange rate risk and the impact of regret on trade. Open Economies Review 26, 109-119.

Loomes, G., 1988. Further evidence of the impact of regret and disappointment in choice under uncertainty. Economica 55, 47-62. 
Loomes, G., Starmer, C., Sugden, R., 1992. Are preferences monotonic-testing some predictions of regret theory. Economica 59, 17-33.

Loomes, G., Sugden, R., 1982. Regret theory: an alternative theory of rational choice under uncertainty. Economic Journal 92, 805-824.

Loomes, G., Sugden, R., 1987. Testing for regret and disappointment in choice under uncertainty. Economic Journal 97, 118-129.

Muermann, A., Mitchell, O., Volkman, J., 2006. Regret, portfolio choice and guarantee in defined contribution schemes. Insurance: Mathematics and Economics 39, 219-229.

Paroush, J., Venezia, I., 1979. On the theory of the competitive firm with a utility function defined on profits and regret. European Economic Review 12, 193-202.

Quiggin, J., 1994. Regret theory with general choice sets. Journal of Risk and Uncertainty $8,153-165$.

Sandmo, A., 1971. On the theory of the competitive firm under price uncertainty. American Economic Review 61, 65-73.

Starmer, C., Sugden, R., 1993. Testing for juxtaposition and event-splitting effects. Journal of Risk and Uncertainty 6, 235-254.

Sugden, R., 1993. An axiomatic foundation of regret. Journal of Economic Theory 60, $159-180$.

Wong, K. P., 2011. Regret theory and the banking firm: the optimal bank interest margin. Economic Modelling 28, 2483-2487.

Wong, K. P., 2012. Production and insurance under regret aversion. Economic Modelling $29,1154-1160$.

Wong, K. P., 2014. Regret theory of the competitive firm. Economic Modelling 36, 172-175.

Wong, K. P., 2015. A regret theory of capital structure. Finance Research Letters 12, $48-57$. 